\title{
BOUNDS ON THE EXPECTED SIZE OF THE MAXIMUM AGREEMENT SUBTREE
}

\author{
DANIEL IRVING BERNSTEIN*, LAM SI TUNG HO ${ }^{\dagger}$, COLBY LONG*, MIKE STEEL ${ }^{\ddagger}$, \\ KATHERINE ST. JOHN ${ }^{\S}$, AND SETH SULLIVANT*
}

Key words. Random trees, agreement subtrees, Yule-Harding distribution.

\begin{abstract}
We prove lower bounds on the expected size of the maximum agreement subtree of two random binary phylogenetic trees under both the uniform distribution and Yule-Harding distribution and prove upper bounds under the Yule-Harding distribution. This positively answers a question posed in earlier work. Determining tight upper and lower bounds remains an open problem.
\end{abstract}

1. Introduction. Leaf-labelled trees are a canonical model for evolutionary histories of sets of species [10]. Let $T_{1}$ and $T_{2}$ be two trees with the same set of leaf labels $X$ (interior vertices are unlabelled). Following [10], a rooted tree is a tree that has exactly one distinguished vertex called the root. A subset $S \subseteq X$ yields an agreement subtree of $T_{1}$ and $T_{2}$ if $\left.T_{1}\right|_{S}=\left.T_{2}\right|_{S}$ where for a tree $T,\left.T\right|_{S}$ is the tree restricted to the leaf label set $S$ and is obtained by supressing all vertices of degree 2 (excepting the root, if $T_{1}$ and $T_{2}$ are rooted). A maximum agreement subtree is a subtree that is an agreement subtree of the maximal size in $T_{1}$ and $T_{2}$ (see Figure 1.1). We note that there might be multiple maximum agreement subtrees for the pair $T_{1}$ and $T_{2}$. Let $\operatorname{MAST}\left(T_{1}, T_{2}\right)$ denote the number of leaves of a maximum agreement subtree of $T_{1}$ and $T_{2}$, which can be computed in polynomial time in $|X|$ [13].

Let $T_{1}$ and $T_{2}$ be two unrooted binary trees with $n$ leaves. It is known that $\operatorname{MAST}\left(T_{1}, T_{2}\right)=\Omega(\sqrt{\log n})$ for any pair of trees [9], and there is always a pair of trees $T_{1}$ and $T_{2}$ such that $\operatorname{MAST}\left(T_{1}, T_{2}\right)=O(\log n)$. Closing the gap on this worst case behaviour is a lingering open problem. This worst case behaviour is quite different if the two input trees are rooted (for rooted trees any agreement subtree is also required to respect the induced rooting); in this case, it is easily seen that, for any $n \geq 2$ there is always a pair of trees $T_{1}$ and $T_{2}$ such that $\operatorname{MAST}\left(T_{1}, T_{2}\right)=2$.

Of practical interest is to understand what the expected size of the maximum agreement subtree when $T_{1}$ and $T_{2}$ are drawn from a suitable distribution on the set of all binary trees. For example, de Vienne, Giraud, and Martin [4] proposed using the maximum agreement subtree as a measure of the congruence between two trees. Understanding the distribution of this statistic can be used in hypothesis tests of the null hypothesis that the two trees were generated at random [7]. For example, the deviation from the null hypothesis between a host tree and a parasite tree could be used as evidence of co-speciation [6].

The mathematical study of the distribution of the size of the maximum agreement subtree was initiated in the work of Bryant, McKenzie, and Steel [2]. They specifically focused on the expectation $f_{u}(n)=\mathbb{E}\left[\operatorname{MAST}\left(T_{1}, T_{2}\right)\right]$ and $f_{Y H}(n)=\mathbb{E}\left[\operatorname{MAST}\left(T_{1}, T_{2}\right)\right]$ where in first case the trees are drawn independently from the uniform distribution

\footnotetext{
*Department of Mathematics, North Carolina State University, Raleigh, NC, USA; \{dibernst, celong2, smsulli2\}@ncsu.edu.

$\dagger$ Department of Biostatistics, University of California, Los Angeles, CA, USA; lamho@ucla.edu

${ }^{\ddagger}$ Biomathematics Research Centre, University of Canterbury, Christchurch, New Zealand mike.steel@canterbury.ac.nz

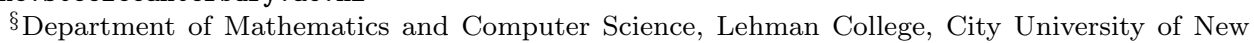
York, Bronx, NY, USA; katherine.stjohn@lehman.cuny.edu; Department of Invertebrate Zoology, American Museum of Natural History, New York, NY, USA.
} 

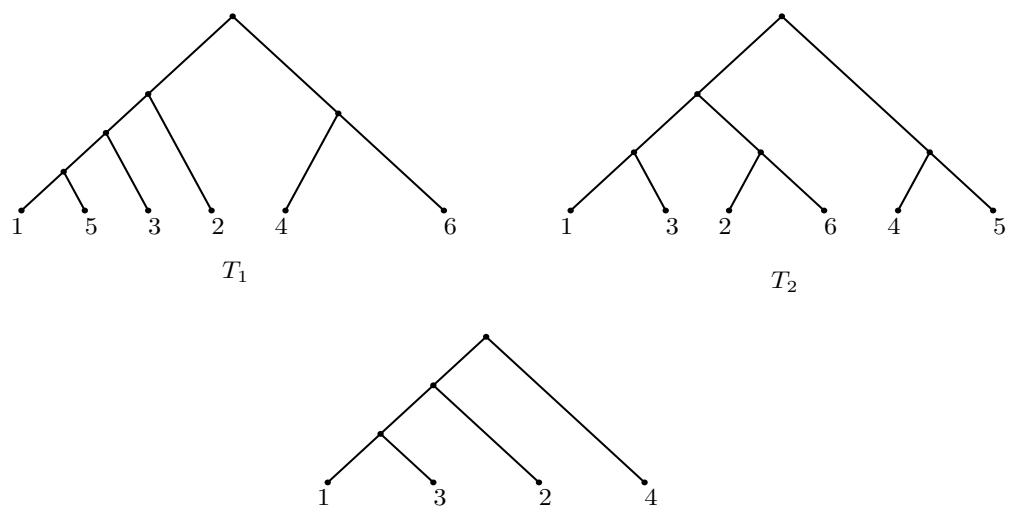

FIG. 1.1. Two rooted phylogenetic trees, $T_{1}$ and $T_{2}$, on 6 leaves and a maximum agreement subtree $(M A S T)$ for $T_{1}$ and $T_{2}$. The MAST illustrated is a caterpillar with leaves encoding the subsequence, $(1,3,2,4)$.

on rooted binary phylogenetic trees with $n$ leaves, and the second case, from the Yule-Harding distribution on rooted binary phylogenetic trees with $n$ leaves (for a formal definition of this distribution, see [10], Section 2.5). Their simulations, for trees with up to $n=1024$ leaves, suggest that under both the uniform distribution on binary trees and the Yule-Harding distribution [5], the expected size of the maximum agreement subtree is of order $\Theta\left(n^{a}\right)$ with $a \approx 1 / 2$, and they also proved that $f_{u}(n)=$ $O\left(n^{1 / 2}\right)$.

In the special case when both random trees are caterpillar trees, finding the maximum agreement subtree is essentially equivalent to finding the longest increasing subsequence in a random permutation. This problem has a long history, and it is well-known that the expected size of the longest increasing subsequence is asymptotically $2 \sqrt{n}$, and that the (appropriately rescaled) distribution of the longest increasing subsequence is the Tracy-Widom distribution (see [1] for a survey of results). The distribution of the maximum agreement subtree is a natural extension of the longest increasing subsequence problem to trees.

Bryant, McKenzie, and Steel [2] posed the question, and again suggested the problem at the 2007 Newton Institute program on Phylogenetics, of finding any exponent $a>0$ such that $f_{u}(n)=\Omega\left(n^{a}\right)$ or $f_{Y H}(n)=\Omega\left(n^{a}\right)$. The main results of this note are, for rooted binary trees, to derive the conjectured (power law type) lower bounds for the expected size of the maximum agreement subtree for both the uniform and YuleHarding distributions, and an upper bound of the form $O\left(n^{1 / 2}\right)$ for the Yule-Harding distribution.

Note that the uniform and Yule-Harding distribution satisfy two fundamental properties, namely exchangeability and sampling consistency. Exchangeability means that if two trees $T_{1}$ and $T_{2}$ differ only by a permutation of the leaves, then $\mathbb{P}_{s}\left(T_{1}\right)=$ $\mathbb{P}_{s}\left(T_{2}\right)$. Sampling consistency is the condition that for any subset $S$ of $X$ if we generate a rooted binary tree $T$ on leaf set $X$ under either model (uniform or Yule-Harding) then the induced tree $\left.T\right|_{S}$ is described by the same model (uniform or Yule-Harding, respectively). For further details see [2].

2. Uniform Trees. To show our lower bound results for rooted binary trees chosen from a uniform distribution, we rely on classical results on the expected largest increasing subsequence in a random permutation of numbers. For trees of size $n$ 
(both unrooted and rooted) we show that the expected length of a caterpillar subtree is $\Omega(\sqrt{n})$. We can then show that for two trees $T_{1}, T_{2}$ chosen independently and uniformly at random from $R B(n)$ there is a subset, $S^{\prime} \subseteq[n]$ of $\Omega\left(n^{1 / 4}\right)$ leaves which induce rooted caterpillars of $T_{1}$ and $T_{2}$. Restricting to this subset $S^{\prime}$, we can view $\left.T_{1}\right|_{S^{\prime}}$ and $\left.T_{2}\right|_{S^{\prime}}$ as permutations of the elements of $S^{\prime}$ and apply the classical results of Aldous and Diaconis [1] to yield a common subsequence of length $\left|S^{\prime}\right|^{1 / 2}=\Omega\left(\left(n^{1 / 4}\right)^{1 / 2}\right)=\Omega\left(n^{1 / 8}\right)$.

Let $R B(n)$ denote the set of rooted binary phylogenetic trees with leaf label set $[n]:=\{1,2,3, \ldots, n\}$. Similarly, $B(n)$ denotes the set of unrooted binary phylogenetic trees with leaf label set $[n]$. Note that $|R B(n)|=(2 n-3) ! !=1 \times 3 \times 5 \times \cdots \times(2 n-3)$, and $b(n)=|B(n)|=(2 n-5)$ !!. In this section, we consider the uniform distribution on $R B(n)$, and the function $f_{u}(n)=\mathbb{E}\left[\operatorname{MAST}\left(T_{1}, T_{2}\right)\right]$ where $T_{1}$ and $T_{2}$ are generated uniformly and independently from $R B(n)$.

Theorem 2.1. For any $\lambda<2^{1 / 4}\left(1-\frac{e^{-1 / 4}}{2}\right)^{2} \approx .443$ there is a value $m$ so that, for all $n \geq m$,

$$
f_{u}(n) \geq \lambda n^{1 / 8}
$$

Let $T$ be an unrooted binary tree with leaves labeled by $[n]$. Then for $i, j \in[n]$, $d_{n}(i, j)$ denotes the number of edges on the unique path from leaf $i$ to leaf $j$.

Proposition 2.2. Let $T$ selected uniformly at random from $B(n)$. For leaves $i, j \in[n], i \neq j$, the probability that $d_{n}(i, j)=m$ is:

$$
\mathbb{P}\left(d_{n}(i, j)=m\right)=\frac{(n-2) !}{(2 n-4) !} \cdot \frac{2^{m-1}(m-1)(2 n-m-4) !}{(n-m-1) !} .
$$

Proof. Let $D(m, n)$ denote the number of $[n]$-trees where $d_{n}(i, j)=m$. If $T$ is an unrooted $[n]$-tree with $d_{n}(i, j)=m$, then we can draw $T$ as follows:

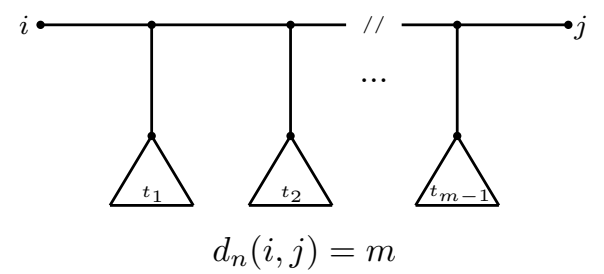

thus giving us a bijection between the set of trees where $d_{n}(i, j)=m$, and the set of ordered forests consisting of rooted binary trees on $n-2$ leaves (as noted by [12] for a different calculation). Now, the set of ordered forests on $m-1$ rooted binary trees and $n-2$ leaves is just $(m-1) ! N(n-2, m-1)$ where $N(r, k)$ is the number of unordered forests of $k$ rooted binary trees and $r$ leaves. Now, $N(r, k)=\frac{(2 r-k-1) !}{(r-k) !(k-1) ! 2^{r-k}}$ for $r \geq k$ and 0 if $r<k$, as stated in Lemma 4 of [3]. This result can be derived by observing that:

$$
N(r, k)=r ! \cdot\left[x^{r}\right] B(x)^{k},
$$

where $B(x)=1-\sqrt{1-2 x}$ is the exponential generating function for the number of rooted binary trees on $k$ non-root leaves, and applying the Lagrange inversion formula, 
together with the identity $B(x)=x\left(1-\frac{1}{2} B(x)\right)^{-1}$, to determine the RHS of (2.1) (for further details, see [10], Section 2.8). This gives the following expression for $D(m, n)$ :

$$
D(m, n)=\frac{(m-1) \cdot(2 n-m-4) !}{2^{n-m-1} \cdot(n-m-1) !} .
$$

Dividing the above by $b(n)=(2 n-5) ! !=\frac{(2 n-4) !}{(n-2) ! \cdot 2^{n-2}}$ gives the desired formula for $P\left(d_{n}(i, j)=m\right)$.

Using Proposition 2.2, we calculate the probability that the path length between two leaves, $i$ and $j$ exceeds $\sqrt{n}$ :

LeMma 2.3. $\lim _{n \rightarrow \infty} \mathbb{P}\left(d_{n}(i, j) \geq \sqrt{n}\right) \geq\left(1-\frac{e^{-1 / 4}}{2}\right)=c \approx 0.61$.

Proof. For a fixed $n$,

$\mathbb{P}\left(d_{n}(i, j)=m+1\right)-\mathbb{P}\left(d_{n}(i, j)=m\right)=\frac{2^{m-1}(2 n-m-5) !(n-2) !}{(n-m-1) !(2 n-4) !}\left(-m^{2}+m+2 n-4\right)$,

which is positive whenever $m \leq \sqrt{2 n}$. Therefore, we have,

$$
\begin{aligned}
\mathbb{P}\left(d_{n}(i, j)<\sqrt{n}\right) & =\sum_{m=1}^{\lceil\sqrt{n}-1\rceil} \mathbb{P}\left(d_{n}(i, j)=m\right) \\
& \leq \sqrt{n}\left(\mathbb{P}\left(d_{n}(i, j)=\sqrt{n}\right) .\right.
\end{aligned}
$$

Using Stirling's approximation for all factorials we have:

$$
\begin{aligned}
\sqrt{n}\left(\mathbb{P}\left(d_{n}(i, j)=\sqrt{n}\right)\right. & \sim \frac{e}{2} \frac{n 2^{\sqrt{n}}(n-2)^{n-2}(2 n-\sqrt{n}-4)^{2 n-\sqrt{n}-4}}{(2 n-4)^{2 n-4}(n-\sqrt{n}-1)^{n-\sqrt{n}-1}} \\
& \sim \frac{e}{2}\left(\frac{2 n-\sqrt{n}-4}{2 n-4}\right)^{2 n}\left(\frac{n-2}{n-\sqrt{n}-1}\right)^{n}\left(\frac{2 n-2 \sqrt{n}-2}{2 n-\sqrt{n}-4}\right)^{\sqrt{n}} \\
& \sim \frac{e}{2}\left(1-\frac{3 n-8}{4(n-2)(n-\sqrt{n}-1)}\right)^{n}\left(1-\frac{\sqrt{n}-2}{2 n-\sqrt{n}-4}\right)^{\sqrt{n}} \\
& \sim \frac{e}{2} e^{-3 / 4} e^{-1 / 2}=\frac{e^{-1 / 4}}{2} .
\end{aligned}
$$

Hence, $\lim _{n \rightarrow \infty} \mathbb{P}\left(d_{n}(i, j)<\sqrt{n}\right) \leq \frac{e^{-1 / 4}}{2}$.

Since $\mathbb{P}\left(d_{n}(i, j) \geq \sqrt{n}\right)=1-P\left(d_{n}(i, j)<\sqrt{n}\right)$, we have

$$
\lim _{n \rightarrow \infty} \mathbb{P}\left(d_{n}(i, j) \geq \sqrt{n}\right) \geq\left(1-\frac{e^{-1 / 4}}{2}\right) .
$$

Recall that a rooted caterpillar on $n$ leaves is any rooted binary phylogenetic tree for which the induced subtree on the interior vertices forms a path graph with the root at one end of the path. We show that two trees, chosen uniformly and independently from rooted binary trees on $n$ leaves, have a common rooted caterpillar of height at least $\Omega\left(n^{1 / 4}\right)$ leaves:

LEMma 2.4. Let $T_{1}, T_{2}$ be rooted $n$-leaf trees chosen uniformly and independently from $R B(n)$. If $n$ is sufficiently large, then with probability greater than $\left(1-\frac{e^{-1 / 4}}{2}\right)^{2}=$ 
$c^{2}$, there exists $S^{\prime} \subset[n]$ with $\left|S^{\prime}\right| \geq \frac{1}{2 \sqrt{2}} n^{1 / 4}$ such that both $\left.T_{1}\right|_{S^{\prime}}$ and $\left.T_{2}\right|_{S^{\prime}}$ are rooted caterpillar trees.

Proof. Let $T_{1}$ and $T_{2}$ be chosen uniformly and independently from $R B(n)$. Choose leaves $i$ and $j$ uniformly at random and temporarily regard $T_{1}$ as an unrooted tree by suppressing the root vertex. If $d_{i j}$ is the distance between $i$ and $j$ in the unrooted tree, then, in the rooted tree, the distance from the root vertex to either $i$ or $j$ must be greater than or equal to $\frac{1}{2} d_{i j}$. Therefore, the probability that $T_{1}$ has height at least $m$ is greater than or equal to the probability that $d_{i j} \geq 2 \mathrm{~m}$. If $T_{1}$ has height $k$ then we can choose $S \subset[n]$ with $|S|=k$ so that $\left.T_{1}\right|_{S}$ is a rooted caterpillar. By Lemma 2.3, the probability of finding $S \subset[n]$ with $|S| \geq \frac{1}{2} n^{1 / 2}$ such that $\left.T_{1}\right|_{S}$ is a rooted caterpillar is greater than $c$. If such an $S$ exists, then since $T_{2}$ was chosen uniformly from $R B(n)$ and independently from $T_{1},\left.T_{2}\right|_{S}$ is a tree chosen uniformly from $R B(|S|)$. Applying Lemma 2.3 again, the probability that there exists an $S^{\prime} \subset[|S|]$ with $\left|S^{\prime}\right| \geq \frac{1}{2} \sqrt{|S|}$ such that $\left.T_{2}\right|_{S^{\prime}}$ is a rooted caterpillar tree is also greater than $c$. Since the restriction of a rooted caterpillar is a rooted caterpillar the result follows.

Now let $\left(T_{1}, T_{2}\right)$ be a pair of trees satisfying the conditions of Lemma 2.4. Select the set $S^{\prime}$ with $\left|S^{\prime}\right|=q(n):=\left\lfloor\frac{1}{2 \sqrt{2}} n^{1 / 4}\right\rfloor$ and relabel the leaves of both $T_{1}$ and $T_{2}$ so that when drawn with the leaf vertex adjacent to the root on the left, the leaf labels of $\left.T_{1}\right|_{S^{\prime}}$ increase from left to right. Draw $T_{2}$ in the same way picking either representation for the leaves of the cherry in $T_{2}$ with equal probability. The order of the leaves of $T_{2}$ gives a permutation uniformly chosen from the set of permutations of $[q]$. From Aldous and Diaconis [1], we have:

THEOREM 2.5. [1, Theorem 2] Let $\pi_{n}$ be a uniform random permutation of $[n]$. Define the integer valued random variable $L_{n}:=l\left(\pi_{n}\right)$ where $l(\pi)$ is the length of the longest increasing subsequence of $\pi$. Then $\mathbb{E}\left[L_{n}\right] \sim 2 n^{1 / 2}$ as $n \rightarrow \infty$.

Observe that if $\sigma$ is the permutation of $[q(n)]$ given by $\left.T_{2}\right|_{S^{\prime}}$ and $s(\sigma)$ is the set of elements of an increasing subsequence of $\sigma$, then $\left.T_{1}\right|_{s(\sigma)}=\left.T_{2}\right|_{s(\sigma)}$ implying $M\left(T_{1}, T_{2}\right) \geq l(\sigma)$. In other words, $\mathbb{P}\left(\operatorname{MAST}\left(T_{1}, T_{2}\right)=i\right)$ is at least the product of the probability that $T_{1}$ and $T_{2}$ restrict to a rooted caterpillar of size $q(n)$ and $\mathbb{P}\left(L_{q(n)}=i\right)$. This is the key observation that will allow us to prove Theorem 2.1.

Proof. [of Theorem 2.1]: By Lemma 2.3 and Theorem 2.5 and the observations above, as $n \rightarrow \infty$

$$
\begin{aligned}
f_{u}(n) & >\sum_{i=1}^{q(n)} i \mathbb{P}\left(M\left(T_{1}, T_{2}\right)=i\right) \\
& >\sum_{i=1}^{q(n)} i\left(c^{2} \mathbb{P}\left(L_{q(n)}=i\right)\right) \\
& =c^{2} \mathbb{E}\left[L_{q(n)}\right] . \\
& \sim 2^{1 / 4} c^{2} n^{1 / 8}
\end{aligned}
$$

3. Yule-Harding Trees: Lower Bounds. In this section we derive our lower bounds on the expected size of the maximum agreement subtree under the YuleHarding distribution [5]. The Yule-Harding distribution is a probability distribution on the set of rooted binary trees that is defined in a constructive manner, by building up a tree on $n$ leaves by successively adjoining leaves. A Yule-Harding tree on $n$ leaves is obtained from a Yule-Harding tree on $n-1$ leaves by choosing a leaf uniformly at 
randomly and branching that leaf into two new leaves. Leaf labels for the $(n-1)$-leaf tree are chosen as a uniformly random subset of size $n-1$ from $[n]$. Let $f_{Y H}(n)$ denote the expected size of the maximum-agreement subtree between two trees from $R B(n)$ sampled independently from the Yule-Harding distribution.

THEOREM 3.1. Let a be the unique positive root of the equation $2^{2-a}=(a+$ 1) $(a+2)$ (approximately $a=.344184 \ldots)$. Then for any $\epsilon>0, f_{Y H}(n)=\Omega\left(n^{a-\epsilon}\right)$. We abbreviate $f_{Y H}(n)=f(n)$ in the arguments below. We first establish a number of preliminary results that are needed for the proof of Theorem 3.1.

Lemma 3.2. Let $b \geq 0$. Then

$$
\frac{1}{b+1} k^{b+1} \leq \sum_{i=1}^{k} i^{b} \leq \frac{1}{b+1}(k+1)^{b+1} .
$$

Proof. This follows by applying the left and right end-point rules for the integral of $\int_{0}^{k} x^{b} d x$.

Next we calculate lower bounds on the overlap of any two splits of $[n]$ (bipartitions of the leaves):

Lemma 3.3. Let $A_{1} \mid B_{1}$ and $A_{2} \mid B_{2}$ be two splits of $[n]$ with $\left|A_{1}\right|=i,\left|A_{2}\right|=j$ and $i \leq j \leq n / 2$. Then either

$$
\begin{aligned}
& \left|A_{1} \cap A_{2}\right| \geq\lceil i / 2\rceil \text { and }\left|B_{1} \cap B_{2}\right| \geq j-\lfloor i / 2\rfloor \text { or } \\
& \left|A_{1} \cap B_{2}\right| \geq\lceil i / 2\rceil \text { and }\left|B_{1} \cap A_{2}\right| \geq j-\lfloor i / 2\rfloor .
\end{aligned}
$$

Proof. Make a $2 \times 2$ matrices whose entries are the four intersection values:

$$
M=\left(\begin{array}{ll}
\left|A_{1} \cap A_{2}\right| & \left|A_{1} \cap B_{2}\right| \\
\left|B_{1} \cap A_{2}\right| & \left|B_{1} \cap B_{2}\right|
\end{array}\right) .
$$

The row sums of $M$ are $i, n-i$ and the column sums are $j, n-j$. So either $M_{11}$ or $M_{12}$ are $\geq\lceil i / 2\rceil$. If $M_{11} \geq\lceil i / 2\rceil$ then $M_{22} \geq n-j-\lfloor i / 2\rfloor \geq j-\lfloor i / 2\rfloor$, since $j \leq n / 2$. If $M_{12} \geq\lceil i / 2\rceil$ then $M_{21} \geq j-\lfloor i / 2\rfloor$.

We can use Lemma 3.3 in a worst case analysis to get lower bounds on $f(n)$.

Lemma 3.4. Let $n=2 k+1$ be odd. Then

$$
f(2 k+1) \geq \frac{8}{(n-1)^{2}} \sum_{1 \leq i<j \leq k}(f(\lceil i / 2\rceil)+f(j-\lfloor i / 2\rfloor))+\frac{8}{(n-1)^{2}} \sum_{1 \leq i \leq k} f(\lceil i / 2\rceil) .
$$

Let $n=2 k$ be even. Then

$$
\begin{aligned}
f(2 k) \geq & \frac{8}{(n-1)^{2}} \sum_{1 \leq i<j<k}(f(\lceil i / 2\rceil)+f(j-\lfloor i / 2\rfloor))+\frac{8}{(n-1)^{2}} \sum_{1 \leq i<k} f(\lceil i / 2\rceil) \\
& +\frac{4}{(n-1)^{2}} \sum_{1 \leq i<k}(f(\lceil i / 2\rceil)+f(k-\lfloor i / 2\rfloor))+\frac{2}{(n-1)^{2}} f(\lceil k / 2\rceil)
\end{aligned}
$$

Proof. For two discrete random variables $X$ and $Y$, the law of total expectation says

$$
\mathbb{E}[X]=\sum_{y} P(Y=y) \mathbb{E}[X \mid Y=y]
$$


We use this identity to get lower bounds on $f(n)$. In particular, we condition on the event that the daughter subtrees of the root in $T_{1}$ and $T_{2}$ have sizes $(i, n-i)$ and $(j, n-j)$ respectively, and so we apply (3.1) with $Y=(i, j)$ and $X=\operatorname{MAST}\left(T_{1}, T_{2}\right)$. Since we are sampling from the Yule-Harding model, the size of the daughter subtrees of $T_{1}$ (and of $T_{2}$ ) follows a uniform distribution (see [11] and also follows directly from basic Pólya Urn theory [8]). Thus, the probability of the conditioning event is $\frac{1}{(n-1)^{2}}$ for any $i, j \in[n-1]$.

By the symmetry of the problem, it suffices to look at pairs $1 \leq i \leq j \leq\lfloor n / 2\rfloor$. This is where the factor of 8 comes from in the various expressions. Once we fix $i$ and $j$, we are restricting to the case where two random Yule-Harding trees have daughter subtrees of the root $A_{1} \mid B_{1}$ and $A_{2} \mid B_{2}$ where $\left|A_{1}\right|=i$ and $\left|A_{2}\right|=j$. To get a lower bound on the conditional expectations, we can reduce to the two subtrees on either $A_{1} \cap A_{2}$ and $B_{1} \cap B_{2}$ or $A_{1} \cap B_{2}$ and $B_{1} \cap A_{2}$, depending on which one satisfies the size requirements from Lemma3.3. On those two induced subtrees we have an expected MAST of size at least $f(\lceil i / 2\rceil)$ and $f(j-\lfloor i / 2\rfloor)$ respectively. Combining those trees together through the common root gives a MAST of expected size $f(\lceil i / 2\rceil)+f(j-$ $\lfloor i / 2\rfloor)$. The formulas follow by analysis by cases with attention to double counting and the boundary cases of $i=j, i<j=n / 2$ and $i=j=n / 2$.

As a direct application, we have by counting the number of occurrences of $f(i)$ :

COROLlary 3.5.

$$
f(n) \geq \frac{8}{(n-1)^{2}} \sum_{i=1}^{\lfloor n / 2\rfloor}(n-2 i) f(i) .
$$

The lower bound for the Yule-Harding case follows by induction on the inequality of Corollary 3.5:

Proof. [of Theorem 3.1] We use the inequality from Corollary 3.5, together with induction. Clearly $f(1)=1 \geq c \times 1^{a}$ for $c=1$. Assume that $f(k) \geq c k^{a}$ for all $k<n$, then we have:

$$
f(n) \geq \frac{8}{(n-1)^{2}} \sum_{i=1}^{\lfloor n / 2\rfloor}(n-2 i) \times c i^{a} .
$$

Applying Lemma 3.2 we deduce

$$
\begin{aligned}
f(n) & \geq \frac{8}{(n-1)^{2}} \cdot c \cdot\left(\frac{n}{a+1}\left\lfloor\frac{n}{2}\right\rfloor^{a+1}-\frac{2}{a+2}\left\lfloor\frac{n}{2}+1\right\rfloor^{a+2}\right) \\
& =\frac{2^{2-a}}{(a+1)(a+2)} \cdot c \cdot \frac{(a+2) n\left(2\left\lfloor\frac{n}{2}\right\rfloor\right)^{a+1}-(a+1)\left(2\left\lfloor\frac{n}{2}+1\right\rfloor\right)^{a+2}}{(n-1)^{2}} .
\end{aligned}
$$

Note that the rightmost expression in the product is asymptotic to $n^{a}$, converging to it from below. In particular, for any $\delta>0$, there exists an $N$ such that for all $n>N$, we have

$$
\frac{(a+2) n\left(2\left\lfloor\frac{n}{2}\right\rfloor\right)^{a+1}-(a+1)\left(2\left\lfloor\frac{n}{2}+1\right\rfloor\right)^{a+2}}{(n-1)^{2}}>(1-\delta) n^{a} .
$$

This yields

$$
f(n) \geq \frac{2^{2-a}}{(a+1)(a+2)}(1-\delta) \times c n^{a} .
$$


To complete the induction we must have

$$
\frac{2^{2-a}}{(a+1)(a+2)}(1-\delta) \geq 1 .
$$

Since we can take $\delta$ arbitrarily small, this completes the result.

Note that in this argument the value of $c$ will depend on $\delta$ (through the interaction with $N$ ). Hence, we cannot use the proof argument to take $\epsilon=0$ in the statement.

REMARK 1. Further modifications to the above argument can be made to get slight improvements on the exponent. For example, when $i$ is very small, instead of taking the subsets of size $\geq\lceil i / 2\rceil$ and $\geq j-\lfloor i / 2\rfloor$, passing to a single subset of size $n-i$ (throwing out the subset of size $i$ ) can yield an improvement in the bounds. That is, if $i$ is small then

$$
f(\lceil i / 2\rceil)+f(j-\lfloor i / 2\rfloor) \leq f(n-i)
$$

when $f(n)=n^{\alpha}$ and $\alpha$ bounded away from 0 . Using this reasoning coupled with the arguments above, we were able to increase the exponent in the theorem to approximately .384 .

4. Yule-Harding Trees: Upper Bounds. In this section, we derive $O\left(n^{1 / 2}\right)$ upper bounds on the expected size of the maximum agreement subtree for any distribution on trees that is exchangeable and satisfies sampling consistency (described at the end of the Introduction) based on ideas from [2]. We just need the crucial Lemma 4.1 from that paper. For a fixed distribution on trees let $\mathbb{P}_{s}(t)$ be the probability of the tree $t$ which has $s$ leaves.

Lemma 4.1. [2, Lemma 4.1] Suppose that phylogenetic trees $T_{1}$ and $T_{2}$ on a leaf set $L$ of size $n$ are randomly generated under a model that satisfies exchangeability and sampling consistency. Then

$$
\mathbb{P}\left[\operatorname{MAST}\left(T_{1}, T_{2}\right) \geq s\right] \leq \psi_{n, s}=\left(\begin{array}{c}
n \\
s
\end{array}\right) \sum_{t \in R B(s)} \mathbb{P}_{s}(t)^{2}
$$

From here, we choose a function $s=g(n)$ so that $\left(\begin{array}{l}n \\ s\end{array}\right) \sum_{t \in R B(s)} \mathbb{P}_{s}(t)^{2}$ tends rapidly to zero with $n$, to deduce that $\mathbb{E}\left[\operatorname{MAST}\left(T_{1}, T_{2}\right)\right]=O(g(n))$.

Proposition 4.2. Let $\mathbb{P}_{s}$ be any exchangeable distribution on rooted binary trees. Then

$$
\sum_{t \in R B(s)} \mathbb{P}_{s}(t)^{2} \leq \frac{2^{s-1}}{s !}
$$

Proof. Let $\mathbb{P}_{s}$ be any exchangeable distribution on rooted binary trees. Note that if $0<x \leq y$ then

$$
x^{2}+y^{2} \leq(x-\epsilon)^{2}+(y+\epsilon)^{2}=x^{2}+y^{2}+2 \epsilon^{2}+2 \epsilon(y-x) .
$$

This implies that if we take $\mathbb{Q}_{s}(t)$ to be the probability distribution that puts zero mass on trees that have a shape different from a tree $t^{\prime}$ that maximizes $\mathbb{P}_{s}\left(t^{\prime}\right)$, we will have

$$
\sum_{t \in R B(s)} \mathbb{P}_{s}(t)^{2} \leq \sum_{t \in R B(s)} \mathbb{Q}_{s}(t)^{2} .
$$


By exchangeability, when $\mathbb{Q}_{s}(t) \neq 0$, it is $\frac{1}{N T(t)}$ where $N T(t)$ is the number of rooted trees with tree shape $t$. Thus,

$$
\sum_{t \in R B(s)} \mathbb{Q}_{s}(t)^{2}=\frac{1}{N T(t)^{2}}+\cdots+\frac{1}{N T(t)^{2}}(N T(t) \text { times })=\frac{1}{N T(t)} .
$$

To maximize this quantity, we choose a tree shape with the fewest number of trees with that tree shape. The number of trees of a given shape $t$ is $s ! / 2^{m}$ where $m$ is the number of internal vertices of $t$ that are symmetry vertices (i.e. vertices of $t$ for which the two daughter subtrees have the same shape [10]). Since a rooted binary tree with $s$ leaves has $s-1$ internal vertices, $m \leq s-1$. Thus, $N T(t) \geq s ! / 2^{s-1}$.

THEOREM 4.3. Let $T_{1}$ and $T_{2}$ be generated from any exchangeable, sampling consistent distribution on rooted binary trees with $n$ leaves. For any $\lambda>e \sqrt{2}$ there is a value $m$ such that, for all $n \geq m$,

$$
\mathbb{E}\left[\operatorname{MAST}\left(T_{1}, T_{2}\right)\right] \leq \lambda \sqrt{n} .
$$

Proof. We explore the asymptotic behaviour of the quantity $\phi_{n, s}=\left(\begin{array}{l}n \\ s\end{array}\right) \frac{2^{s-1}}{s !}$. Using the inequality $\left(\begin{array}{l}n \\ s\end{array}\right) \leq \frac{n^{s}}{s !}$ and Stirling's approximation, we have:

$$
\phi_{n, s} \leq \frac{1}{4 \pi s}\left(\frac{2 e^{2} n}{s^{2}}\right)^{s} \theta(s)
$$

where $\theta(s) \sim 1$. Hence, $\phi_{n, s}$ tends to zero as an exponential function of $n$ as $n \rightarrow \infty$. Since $\phi_{n, s} \geq \psi_{n, s}$ we see that $\mathbb{P}\left[\operatorname{MAST}\left(T_{1}, T_{2}\right)>\lambda \sqrt{n}\right.$ tends to zero as an exponential function of $n$. Since $\operatorname{MAST}\left(T_{1}, T_{2}\right) \leq n$, this implies that $\mathbb{E}\left[\operatorname{MAST}\left(T_{1}, T_{2}\right)\right] \leq \lambda \sqrt{n}$.

Acknowledgments. Work on this project was started at the 2014 NSF-CBMS Workshop on Phylogenetics at Winthrop University, partially supported by the US National Science Foundation (DMS 1346946). Lam Ho was partially supported by the NSF Grant DMS 1264153 and the NIH Grant R01 AI107034. Colby Long was partially supported by the US National Science Foundation (DMS 0954865). Mike Steel was partially supported by the NZ Marsden Fund. Katherine St. John was partially supported by the Simons Foundation. Seth Sullivant was partially supported by the David and Lucille Packard Foundation and the US National Science Foundation (DMS 0954865). Finally, we thank the three anonymous reviewers for their helpful comments concerning an earlier version of this manuscript. 


\section{REFERENCES}

[1] D. Aldous and P. Diaconis. Longest increasing increasing subsequences: From patience sorting to the Baik-Deift-Johansson theorem. Bulletin of the American Mathematical Society, 36(4) (1999):413-432.

[2] D. Bryant, A. McKenzie and M. Steel. The size of a maximum agreement subtree for random binary trees. In BioConsensus (DIMACS Series in discrete mathematics and theoretical computer science), American Mathematical Society 61 (2003):55-65.

[3] M. Carter, M. Hendy, D. Penny, L.A. Székely and N.C. Wormald. On the distribution of lengths of evolutionary trees. SIAM J. Discr. Math. 3(1) (1990):38-47.

[4] D.M. de Vienne, T. Giraud, O. C. Martin. A congruence index for testing topological similarity between trees. Bioinformatics 23(23) (2007):3119-3124

[5] E. F. Harding. The probabilities of rooted tree shapes generated by random bifurcation. Advances in Applied Probability 3(1) (1971):44-77.

[6] E. Jousselin, S. Van Noort, V. Berry, J-Y Rasplus, N. Rønsted, J.C. Erasmus, J.M. Greeff. One fig to bind them all: Host conservativesm in a fig wasp community unraveled by cospeciation analysis among pollinating and nonpollinating fig wasps. Evolution 62(7) (2008):1777-1797.

[7] F.-J. Lapointe, L.J. Rissler, Congruence, consensus, and the comparative phylogeography of codistributed species in California. Am. Nat. 166(2) (2005):290-299.

[8] H. M. Mahmoud. Pólya Urn Models. Chapman and Hall/CRC Press. 2008.

[9] D.M. Martin and B. D. Thatte. The maximum agreement subtree problem. Discrete Appl. Math. 161 (2013):1805-1817.

[10] C. Semple and M. Steel. Phylogenetics. Oxford University Press. 2003.

[11] J. B. Slowinski. Probabilities of n-Trees Under Two Models: A Demonstration that Asymmetrical Interior Nodes are not Improbable. Systematic Zoology 39(1) (1990):89-94

[12] M. A. Steel and D. Penny. Distributions of tree comparison metrics - some new results. Systematic Biology 42 (1993):126-141.

[13] M. A. Steel and T. Warnow. Kaikoura tree theorems: computing the maximum agreement subtree. Information Processing Letters 48 (1993):77-82. 